1

\title{
A Novel Taxon of RNA Viruses Endemic to Planarian Flatworms
}

\author{
Jeffrey Burrows ${ }^{1,2}$, Delphine Depierreux ${ }^{3}$, Max L. Nibert ${ }^{3}$, and Bret J. Pearson ${ }^{1,2,4^{*}}$ \\ ${ }^{1}$ The Hospital for Sick Children, Program in Developmental and Stem Cell Biology, Toronto, ON, \\ Canada, M5G0A4; \\ ${ }^{2}$ University of Toronto, Department of Molecular Genetics, Toronto, ON, Canada; \\ ${ }^{3}$ Department of Microbiology, Harvard Medical School, Boston, MA 02115, USA \\ ${ }^{4}$ Ontario Institute for Cancer Research, Toronto, ON, Canada \\ *Correspondence should be addressed to: Bret Pearson (email: bret.pearson@ sickkids.ca) \\ Keywords: dsRNA virus, evolution, regeneration, Lophotrochozoan, planarians, Schmidtea \\ mediterranea
}




\section{Abstract}

The phylum Platyhelminthes is composed of both parasitic and non-parasitic flatworms. While the parasitic species have drawn attention for their wide effects on human and livestock heath, free-living flatworms, such as freshwater planarians, have become molecular models of regeneration and stem cell biology in the laboratory. However, one aspect of planarian biology that remains understudied is the relationship between host and any endemic viruses. Here we used searches of multiple transcriptomes from Schmidtea mediterranea asexual strain CIW4 and detected a novel, double-stranded RNA (dsRNA) virus, named S. mediterranea tricladivirus (SmedTV), which represents a distinct taxon (proposed new genus) within a larger taxon of monosegmented dsRNA viruses of diverse hosts. Experimental evidence for SmedTV in $S$. mediterranea CIW4 was obtained through whole-mount in situ hybridization (WISH). SmedTV “expression" (detected by both sense and anti-sense probes) was discrete yet variable from worm to worm and cell type to cell type, suggesting a persistent infection. Single-cell RNA sequencing (scRNAseq) further supported that SmedTV expression was low in stem cells, but substantially higher in multiple, though not all, differentiated tissues, with notable neural enrichment. Interestingly, knockdown of SmedTV by RNA-interference resulted in a "cure" of SmedTV after 10 RNAi doses, and expression remained undetectable by WISH even after 90 days. Due to

3 being able to evade host defenses and the endogenous RNAi pathway, we believe SmedTV 4 represents a novel animal model to study host-virus evolution. 


\section{Statement of significance}

46 Planarians are freshwater flatworms and emerging models to study the molecular mechanisms of

47 adult stem cell and regenerative biology. However, they also live in aquatic environments with

48 high amounts of viruses, bacteria, fungi, and protist pathogens. How the planarian immune

49 system copes with all of these is largely unknown and only 2 types of virus have been described.

50 Here we find a novel dsRNA virus, endemic to multiple types of flatworms. We show that it is a

51 persistent infection, and likely transmits from stem cell to differentiated cell in the planarian,

52 while avoiding endogenous RNA-interference machinery and mechanisms used to suppress

53 viruses. We present this as a new model to study host-virus defense and evolution. 


\section{Introduction}

The phylum Platyhelminthes encompasses both parasitic and nonparasitic species of

57 flatworms. Two classes, Cestoda and Trematoda, include globally important parasites of humans

58 (tapeworms and flukes, respectively) and another class, Monogenea, includes parasites of fish

59 (Verneau, Du Preez et al. 2009, Ramm 2017). Planarians fall outside these three classes of

60 parasitic flatworms, and species commonly used as laboratory models are members of order

61 Tricladida. Of these free-living (nonparasitic) species, Schmidtea mediterranea (S. mediterranea)

62 is the most extensively used as a model system for adult tissue regeneration and stem cell

63 biology (Reddien and Sanchez Alvarado 2004).

When manually cut into pieces, planarians fragments can regenerate any lost tissues to

65 become complete animals thanks to a large population of pluripotent stem cells (Newmark and

66 Sanchez Alvarado 2002, Wagner, Wang et al. 2011, Zhu and Pearson 2016). In fact, by

67 employing the simple method of manual division, clonal lines of $S$. mediterranea and other

68 planarians can be serially maintained in research laboratories without the need for sexual

69 reproduction. From a logistical point of view, such conditions would appear to be ideally suited

70 for maintenance of persistent viruses as there would be no need to endure meiosis and the virus

71 could be passed from mother to daughter cells by division without need for de novo infection

72 from the outside. To date the only virus-like elements to be reported in planarians are two related

73 small putative DNA viruses in Girardia tigrina (Rebrikov, Bogdanova et al. 2002, Rebrikov,

74 Bulina et al. 2002), as well as a recent nidovirus that is the largest example of a virus in that

75 family ever described (Saberi, Gulyaeva et al. 2018), suggesting that planarians may be

76 informative hosts for viral evolution. Despite these findings, many questions remain: What

77 species might constitute the normal planarian virome?; What effect might these natural 
pathogens might have on planarian biology? And; what is the co-evolutionary effect on viral biology in the context of planarian immune defenses?

Recent years have seen an explosion of virus discovery, both DNA and RNA viruses, rooted in improving methods for high-throughput sequencing of biologically complex samples (Holmes 2009, Roossinck 2017). For the purposes of this study, we took account of the fact that numerous transcriptomes have been reported for $S$. mediterranea and other planarians, in large part due to the utility of transcriptome studies for identifying key factors in tissue regeneration and development (Solana, Kao et al. 2012, Kao, Felix et al. 2013, Brandl, Moon et al. 2016). We therefore decided to probe these planarian transcriptomes for novel viruses by bioinformatic means. Here we report a new taxon of totivirus-like, monosegmented dsRNA viruses from 5 different species of triclad planarians, including from multiple laboratory lines of asexual $S$. mediterranea. We used in situ hybridization, RNA-interference (RNAi), and other methods to begin to address biological questions about the virus in $S$. mediterranea, including its distribution in different cell types, as described in detail below. These findings set the stage for additional ongoing studies of these novel viruses, their possible effects on different aspects of planarian biology, and viral mechanisms of host immunity avoidance. The findings highlight the fact that published transcriptomes are a powerful resource for virus discovery. We predict that by understanding the mechanisms through which these and other viruses express their gene products and reproduce in planarians will allow for transgenic methods for these animals in the future. 
Methods), uncovered the apparently full-length coding sequences of 5 new species of totiviruslike, nonsegmented dsRNA viruses from 5 respective species of flatworms (phylum proposed names for the 5 new viruses, represented by "TV" for "totivirus" are listed in Table 1.

Features of the plus-strand sequences of these viruses are shown in Fig. 1. They span 114 similar overall lengths (7808-8511 nt) and exhibit a shared coding strategy encompassing three 115 long ORFs. The longest of these ORFs (designated ORF1) is overlapped by both of the other two 116 ORFs: the shortest ORF (designated ORF-s) overlaps the $5^{\prime}$ end of ORF1 and the middle-sized 117 ORF (designated ORF2) overlaps the $3^{\prime}$ end of ORF1. ORF-s is found in the -1 frame relative to 118 ORF1, and translation of these two ORFs seems likely to involve respectively distinct initiation 119 mechanisms since their putative AUG start codons are somewhat closely juxtaposed in their 120 respective reading frames. ORF2, in contrast, is found in the +1 frame relative to ORF1 and seems 121 likely to be translated in fusion with ORF1 via +1 programmed ribosomal frameshifting, with the 122 proposed +1 slippery sequence UUU_C (Firth et al., 2012) (underline indicates codon boundary 123 in the ORF1 frame) apparent in the region of ORF1-ORF2 overlap in 4 of the 5 viruses. Properties 
124 of the deduced proteins P-s and P1 plus fusion protein P1+2 are shown in Table 1 and exhibit

125 strong similarities among the 5 viruses, particularly for P1 and P1+2.

We used the 5 virus sequences illustrated in Fig. 1 as queries for BLASTX searches of the

127 GenBank Nonredundant Protein Sequences (NR) database for dsRNA viruses (taxid 35325). These

128 searches identified two regions of sequence similarities to previously characterized toti-like viruses

129 from animals: a region in the central portion of the queries, encompassed by ORF1, with strongest

130 similarities to the coat protein (CP) of piscine myocarditis virus (PCMV; (Haugland, Mikalsen et

131 al. 2011)) (best E-value, 1.5e-07) and a region in the $3^{\prime}$ half of the queries, encompassed by ORF2,

132 with strongest similarities to the RNA-dependent RNA polymerase (RdRp) of Leptopilina

133 boulardi (parasitoid wasp) toti-like virus (LbTV; (Martinez, Lepetit et al. 2016)) (best E-value

134 1e-12) (Table 2). When the sequences illustrated in Fig. 1 were instead used as queries for

BLASTX searches of the NR database for all viruses (taxid 10239), additional hits were found to

a number of unclassified RNA viruses from a transcriptome study of potential invertebrate hosts

by (Shi, Lin et al. 2016) (Table S1). These unclassified RNA viruses seem likely also to have nonsegmented dsRNA genomes, and indeed most of them are named as "toti-like" viruses.

The Triclad planarian S. mediterranea is the most studied of the apparent hosts of the new

141 available at GenBank (GCA_000181075, GCA_000572305, GCA_000691995, and

142 GCA_002600895.1). Using the SmedTV sequence as query for a Discontiguous MegaBLAST of

143 these genome assemblies failed to identify any significant similarities (E-values, >10), providing

144 evidence that SmedTV derives from an extra-genomic source in $S$. mediterranea, most likely from

145 an actively replicating dsRNA virus. 
Sequence comparisons and phylogenetic analyses

148

149

150

151

152

153

154

155

156

157

158

159

160

We made use of phylogenetic methods to investigate further the relationship between the apparent new flatworm viruses and a larger collection of previously reported toti-like viruses. Representatives of the 5 formally recognized genera of family Totiviridae (Giardiavirus, Leishmaniavirus, Totivirus, Trichomonasvirus, and Victorivirus, each comprising fungal or protozoal viruses) were included in this analysis, as were fish virus PCMV and insect virus LbTV described above. Also included were a number of other toti-like viruses whose RdRps were (i) identified as homologs in BLASTP searches and (ii) also represented in the Reference Sequence (RefSeq) database at NCBI (see Table S2 for all virus names, abbreviations, and RefSeq accession numbers). Following multiple sequence alignments and maximum likelihood phylogenetic analyses of the RdRps of these viruses, results like those shown in Fig. 2 were consistently obtained, indicating that the flatworm viruses constitute a distinct monophyletic clade within the larger collection of toti-like viruses. Branching most closely to the flatworm virus clade $(I)$ in Fig. 2 are three other distinguishable clades of viruses associated with arthropod hosts $(I I-I V)$, one that includes LbTV (III); a distinguishable clade of fish viruses including PCMV (V); and a clade defined by Giardia lamblia virus $(V I)$. The clade of apparent arthropod viruses that branches most closely to the flatworm viruses (II) is notable for having genomes that encompass three long ORFs each, organized comparably to those of the flatworm viruses (ORF-s, ORF1, and ORF2) except that the genome length is somewhat shorter (6618-6969 nt) and that ORF1 (CP) and ORF2 (RdRp) do not overlap (Fig. S1; pairwise alignments in Fig. S2).

\section{SmedTV in different strains of $\mathrm{S}$. mediterranea and validation by amplicon sequencing}

A large number of transcriptome BioProjects with available SRA data for S. mediterranea are available at NCBI (52 as of this writing). We examined these with an effort toward assembling SmedTV sequences from particular well-annotated strains of S. mediterranea, for comparison 
174 purposes such as for tracing S. mediterranea lineages. Toward this end, we generated 11 complete

175 coding sequences for SmedTV from selected BioProjects (registration dates 2011-2017) that have

176 a sufficiently large number of SmedTV-matching reads. All 11 of these SmedTV sequences could

177 be aligned without gaps over a shared 7858-nt region, including the expected three ORFs described

178 above and exhibiting $>99.5 \%$ sequence identity in pairwise comparisons. The numbers of

179 nucleotide mismatches in the pairwise alignments ranged from 0 to 38 (Fig. S3). For example,

180 SmedTV sequences assembled from Pearson lab BioProjects registered in 2012, 2015, and 2017

181 for strain CIW4 were 100\% identical (0 nucleotide mismatches), suggesting genetic stability of

182 the virus within a particular lab colony lineage. Comparing all 11 SmedTV sequences, three main

183 clades appeared to be defined, including two distinguishable clades of the virus from $S$.

184 mediterranea annotated as CIW4 strains from several different labs (Figs. 2 and S3).

Among the 11 strains of $S$. mediterranea used for generating SmedTV sequences deposited

at NCBI, all were annotated as asexual. By examining all BioProjects containing samples

annotated as deriving from sexual strains of $S$. mediterranea (14 BioProjects as of this writing), we found that none were strongly positive for SmedTV-matching reads: 10 of these BioProjects had 0 matching reads, whereas 4 contained only small numbers of SmedTV-matching reads, 3-21.

The transcriptome for the sexual S2F2 strain of S. mediterranea at PlanMine (Smes)(Rozanski, Moon et al. 2019) was also found to lack SmedTV-matching reads. Based on these findings, we conclude that sexual strains of $S$. mediterranea in current use by different labs are apparently not infected with SmedTV. 
S. mediterranea itself. We first performed PCR using SmedTV-specific primers on RNA-derived cDNA either from extensively washed planarians of $S$. mediterranea asexual strain CIW4 or from passaged culture medium from which planarians were excluded. An amplicon of expected size was produced only from the planarian sample, arguing against a contaminating source of SmedTV (Fig. S4). Notably, S. mediterranea sexual strain S2F2 also failed to yield an amplicon (Fig. S4), consistent with the paucity of SmedTV-matching reads in the transcriptomes of sexual $S$. mediterranea strains described above.

Further evidence for the presence of SmedTV in S. mediterranea CIW4 was obtained through whole-mount in situ hybridization (WISH) using riboprobes for detecting SmedTV RNA. In one set of experiments, WISH was performed using dual fluorescent riboprobes to detect either the plus strand (sense, SE) or the minus strand (antisense, AS) of SmedTV genome (Fig. 3A, top row). We found that both strands consistently colocalized in a subset of discrete $S$. mediterranea cells (101/101 SmedTV-positive cells counted), consistent with the presence of RNA representing both strands of the SmedTV genome within each of these cells. The highly punctate staining of SmedTV signal detected by WISH co-localized with a DAPI nuclear counterstain, showing nuclear localization of SmedTV (Fig. 3A, middle row).

Examining the spatial distribution of $S m e d T V$-positive cells within the whole body of $S$. mediterranea CIW4, we found labeled cells to be scattered throughout each worm, though most clearly concentrated in the eye spots and brain lobes (Fig. 3A, bottom row). Interestingly, the distribution of SmedTV-positive cells was similar from worm to worm within the same culture

221 container, but the number of SmedTV-positive cells could be highly variable between worms from 222 different containers. In cultures with "high" levels, there was a notable increase in staining of the 223 head and its associated neural structures. This was quantified in worms from representative "high" 224 and "low" cultures (Fig. S5A), which revealed more than a two-fold difference in the number of 225 SmedTV-positive cells $(p$-value $<0.0001)$ in the "high" worms (mean $=408 \pm 20$ cells per $\mathrm{mm}^{2}, \mathrm{n}$ $226=8)$ vs. the "low" worms (mean $=180 \pm 20$ cells per $\left.\mathrm{mm}^{2}, \mathrm{n}=8\right)$. Moreover, this quantification 
227 is likely an under-representation, since SmedTV-positive cells in the heads of "high" worms were

228 so densely packed as to hinder accurate counting.

\section{Colocalization of SmedTV with neuronal markers in brains and eyes}

231

232

233

234

235

To obtain a more precise demonstration of the nature of the SmedTV-positive cells, we performed double-fluorescence in situ hybridization ( $\mathrm{dFISH})$ on manually generated transverse sections of worm heads. We used riboprobes for detecting the RNA of either SmedTV or the cholinergic neuron marker choline acetyltransferase $(C h A T)($ Cebria, Kudome et al. 2002) (Fig. 3B, top two rows; ChAT expression in these images defines the left brain lobe (dashed outlines), but can also be seen in the peripheral nervous system in the outer edges of the worm. Notably, SmedTV+ cells were found within both of these neuron-rich regions, in some cases colocalizing with $C h A T$. In addition, the numbers of SmedTV+ cells again varied between worms from "high" and "low" cultures, as defined above (Fig. S5B).

As suggested above, SmedTV was also enriched in the eye spots of worms from both "high" and "low" cultures. To quantify this localization, we performed dFISH with SmedTV and the photoreceptor neuron marker Smed-opsin (Fig. 3B, bottom row). SmedTV-positive cells were clearly observed within photoreceptors. Restricting quantification to worms from "low" cultures, we found that similar levels of SmedTV-positive photoreceptors were present in both eyes (Fig $\mathrm{S} 5 \mathrm{C}$, right, mean $=12.3 \pm 1.7$; left, mean $=11.6 \pm 1.6 ; \mathrm{n}=7$ for both eyes $)$.

Of note, brain and eyes are differentiated cell types. Previously published high-throughput RNA sequencing data demonstrated that there was a low level of SmedTV detection in the stem cell compartment ("X1" population; 1.3 reads per million (RPM)) compared to differentiated tissues 19.7 RPM) (Labbe, Irimia et al. 2012). Consistent with this, SmedTV transcript was not 
251 detected in piwi-1 expressing stem cells by dFISH (Fig. 3C, green and magenta respectively). Two

252 published single-cell RNA sequencing (scRNAseq) datasets, from two different laboratories

253 (Wurtzel, Cote et al. 2015, Molinaro and Pearson 2016), further supported the observation that

254 SmedTV expression was low in stem cells and higher in differentiated tissues, particularly neural

255 cell types (Fig. 3D).

256

257 SmedTV expression dynamics during regeneration and interrogation of possible host defenses 
273 expression (Fig. S6A top). Similarly, when stem cells were ablated by ionizing irradiation, no

274 observable increase SmedTV expression was noted (Fig. S6A bottom).

We next pursued RNAi knockdown of two additional candidate regulators, retinoic acid-

276 inducible gene sRNA, RIG-1, and nuclease encoding gene nucl/endoG. $R I G-1$ encodes for a

277 dsRNA helicase enzyme is essential for recognition and infection control of many RNA viruses

278 (Kell and Gale 2015). nuc1/endoG is an endonuclease that has been shown to suppress dsRNA

279 virus replication in yeast (Liu and Dieckmann 1989). However, we observed no difference in the

280 number of SmedTV positive cells by WISH in either RIG-1 or nucl/endoG knockdown worms

281 relative to controls (Fig S6B-C).

Next we tested whether SmedTV was specifically de-repressed in dying cells. To test this we combined FISH and TUNEL staining to determine if dying cells were also SmedTV positive.

Only a single double positive cell was identified in 75 TUNEL, thus, we concluded that the apoptotic state of a cell is not related to SmedTV expression (Fig. S6D). 
infection. To test these possibilities, we injected a sonicated homogenate from a "high" SmedTV-

Ten such injections failed to result in transmission of the virus as seen by WISH analysis of

SmedTV expression (Fig. 4C). Moreover, no evidence of viral particles were observed by EM

SmedTV+ cells (50/60 eyes in 30 worms scored). Taken together these results suggest to us that

to organism and that natural transmission may occur instead only vertically by intracellular means, from dividing stem cells to their daughters.

\section{Discussion}

In this study, we identify a dsRNA totivirus-like element from 5 species of Platyhelminth 
Interestingly, the virus could also be "cured" by RNAi, which raises questions about how the virus normally propagates and evades endogenous RNAi machinery (Fig. 4). Finally, we present

321 evidence that the virus is likely not suppressed by PIWI-dependent piRNA silencing, nor the RIG-

3221 anti-viral recognition system (Fig S6A,B).

323

\section{Nature of the SmedTV infection and remaining questions}

Despite our analyses, many interesting questions remain regarding SmedTV function, and propagation: 1) How does the virus avoid detection by host? 2) Why is "expression" low/repressed in certain cell types and tissues (e.g. stem cells and blastemas) and permitted/high in others (neural tissues)? 3) How is the virus transmitted from cell to cell considering that all tissues turnover and regenerate? 4) Can the virus be used to make elusive transgenic planarians?

Using transcriptome data, we found widespread, persistent infection of asexual $S$. mediterranea with SmedTV across the world, yet there is no evidence for illness in the $S$. mediterranea CIW4 colony used in this study. This suggests a mutualistic or commensal relationship between virus and host. However, planarians have endogenous mechanisms to suppress exogenous elements, such as PIWI-associated small RNAs, as well as the well know RIG-1 antiviral program (Kell and Gale 2015, Ozata, Gainetdinov et al. 2019). We tested whether either of these may be responsible for repressing viral expressing in stem cells, but could not find experimental evidence to support this hypothesis. However, we found that the SmedTV is susceptible to RNAi itself, which can "cure" animals of the virus. How SmedTV evades and does not trigger the RNAi pathway is interesting. Perhaps part of this mechanism is the clear subcellular localization to the nucleus (Fig. 3). Another possibility is the presumed poly-adenylation of the viral genome may play a role in protecting it from degradation. In either case, however, the virus 
341 is still degraded when a systemic RNAi response to the virus is triggered through normal RNAi

342 administration.

Perhaps part of the host-evasion strategy, and not triggering a systemic RNAi response has

344 to do with the differential "expression" of the virus in certain cell types. We observed that SmedTV

345 could not be readily detected in stem cells or new blastema tissue, but was very highly detected in

346 neural tissues. It is unclear why neurons specifically allow for an environment of SmedTV

347 expression/replication, but even more interesting is whether there is an active neural infection

348 between cells, or whether neurons inherit low levels of virus from their parental stem cells. We

349 tested whether infectious virions are produced by lysing cells from "high" viral animals and

injecting those homogenates into multiple strains of uninfected animals over 10 injection days.

351 We never observed any subsequent active infection, and concluded that the virus is no longer

352 actively infectious. Thus, we believe that transmission from parental stem cell to differentiated cell

353 is the mode of viral propagation, although this does not explain why the viral expression is largely

354 neural specific, or why the viral detection in stem cells is so low by bulk RNAseq (1.25 RPM)

355 (Labbe, Irimia et al. 2012).

We believe that ultimately, understanding how foreign, selfish, endogenous nucleic acid

357 elements propagate in planarians will lead to technology development in the area of transgenics,

358 which do not currently exist in planarians; even in terms of transient expression of a fluorophore.

359 It is interesting to speculate that if the viral coat protein of SmedTV were replaced with a marker

360 enzyme or fluorophore, this maybe be electroporated back into the animals and maintained in a

361 similar fashion as the endogenous SmedTV. If we can find the mechanisms by which $\operatorname{SmedTV}$ is

362 suppressed in stem cells or blastemas, it may be possible to get expression in these cell types when 
transposable elements that are described in planarians, the more potential tools we have for the eventual creation of transgenic tools.

\section{Materials and Methods}

Planarian maintenance, irradiation and injections

Asexual clonal populations of $S$. mediterranea (strain CIW4) and S. polychroa were maintained

371 under standard laboratory conditions, as previously described (Zhu, Hallows et al. 2015). For

372 irradiation experiments (Fig. S6A bottom), planarians were exposed to 60 Gy of $\gamma$-irradiation from

373 a Cs-137 source, Gammacell@ 40 extractor irradiator (Best Theratronics). SmedTV-positive worm

374 homogenate was generated by sonicating pooled CIW4 S. mediterranea worms in physiological

375 salt $(150 \mathrm{mM} \mathrm{NaCl}, 10 \mathrm{mM} \mathrm{MgCl} 2,10 \mathrm{mM}$ Tris $\mathrm{pH} 7.5)$ with 4 pluses at power level 3 on a Fisher

376 Scientific (Hampton, NH) Model 100 Sonic Disemembrator. The homogenate was cooled on ice

377 for 10 seconds between pulses and gently spun afterwards to pellet any large debris. The resulting

378 supernatant was injected into the mesenchyme of $S$. polychroa using a Drummond Scientific

379 (Broomall, PA) Nanoject injector mounted on micromanipulator. Worms were immobilized using

381 injected with multiple (2-6) $32.2 \mathrm{nl}$ pulses of homogenate until gut branches were filled. Worms

382 were injected 10 times total with 2-3 days between injections. Injection needles were made from

383 glass capillaries (30-0050 GC120TF-10, Harvard Apparatus Limited (Holliston, MA)) using a P-

38497 Flaming/Brown Micropipette Puller (Sutter Instruments, Novato, CA). 
dsRNA-expressing E. coli cultures were prepared using pT4P clones, mixed with homogenized calf liver, and fed to animals as previously described (Zhu, Hallows et al. 2015). Unless otherwise stated, animals were feed every 3 days and rinsed each day between feedings. The number of feeds (F) and days (D) after at which the phenotypes were analyzed varied for each experimental treatment and are listed in the corresponding figures. For the piwi-1, 2 and 3 knock down equal amounts of dsRNA-expressing bacterial cultures were combined to prepare RNAi food (Gurley, Rink et al. 2008). For the SmedTV RNAi experiments, worms were subjected to 2 rounds of head and tail amputation to promote tissue turnover after 4 and 7 feeds. Trunk fragments were given 1 week to regenerate prior to recommencement of the RNAi feedings. In all cases, an RNAi vector with GFP coding sequence was used as a negative control.

\section{WISH, dFISH and TUNEL}

WISH and dFISH were performed as previously described (Pearson, Eisenhoffer et al. 2009, Lauter, Soll et al. 2011, Currie, Brown et al. 2016). Riboprobes were prepared from pT4P clones, and imaged as described above. SmedTV specific probes were designed to target both 5 (SmedTV
(5')-SE:
AAGGTATGACCCAGCCACTG,
SmedTV
(5')-AS:

ATAACTTCAGGCGCATCACC) and 3` (SmedTV (3`)-SE: ATGAAGGGCAATCCTCACAG, SmedTV (3`)-AS: GCTATAACGCAAAGGCAACAC) ends of the SmedTV transcript. For the SmedTV dFISH co-localization analysis SE probes were generated from the 5 end of SmedTV genome and AS from the 3 end. Whole mount TUNEL was performed as previously described (Pellettieri and Alvarado 2007).

\section{Microscopy and image acquisition, processing and analysis}


410 Colorimetric WISH stains were imaged on a Leica M165 FC fluorescent dissecting microscope

411 with a Leica DFC7000 T digital camera. Photographs of whole animals were obtained with an

412 Olympus SZX16 microscope equipped with a DP72 digital camera. dFISH results (whole animal

413 and cross sections) were photographed with a Quorum Spinning Disk Confocal 2 (Olympus IX81

414 microscope and Hamamatsu C9100-13 EM-CCD camera). Raw images were captured using

415 Perkin Elmer Volocity (confocal) software and stitched together for whole-animal images. Images

416 were post-processed in Adobe Photoshop and figures assembled in Adobe Illustrator. Linear

417 adjustments (brightness and contrast) were made for images of animals labeled by WISH, FISH,

$418 \mathrm{dFISH}$, and TUNEL in order to best represent actual results. These adjustments were identical

419 within a given experiment where comparisons were drawn between conditions. Cell counts

420 (SmedTV expressing cells (alone or doubled with SmedTV-SE, ChAT, opsin or TUNEL) were

421 quantified using freely available ImageJ software (http://rsb.info.nih.gov/ij/). Graphs and statistic

422 were generated using GraphPad Prism software (GraphPad Software, Inc.). Significance was

423 determined by a 2-tailed Student's t-test with equal or unequal variance as specified. To eliminate

424 any bias due to difference in detection threshold with different development techniques, only cells

425 that were completely encompassed within Z-projections were counted for SmedTV SE and AS co-

426 localization analysis. 


\section{$427 \quad$ Figures}

\section{$428 \quad$ Figure 1}
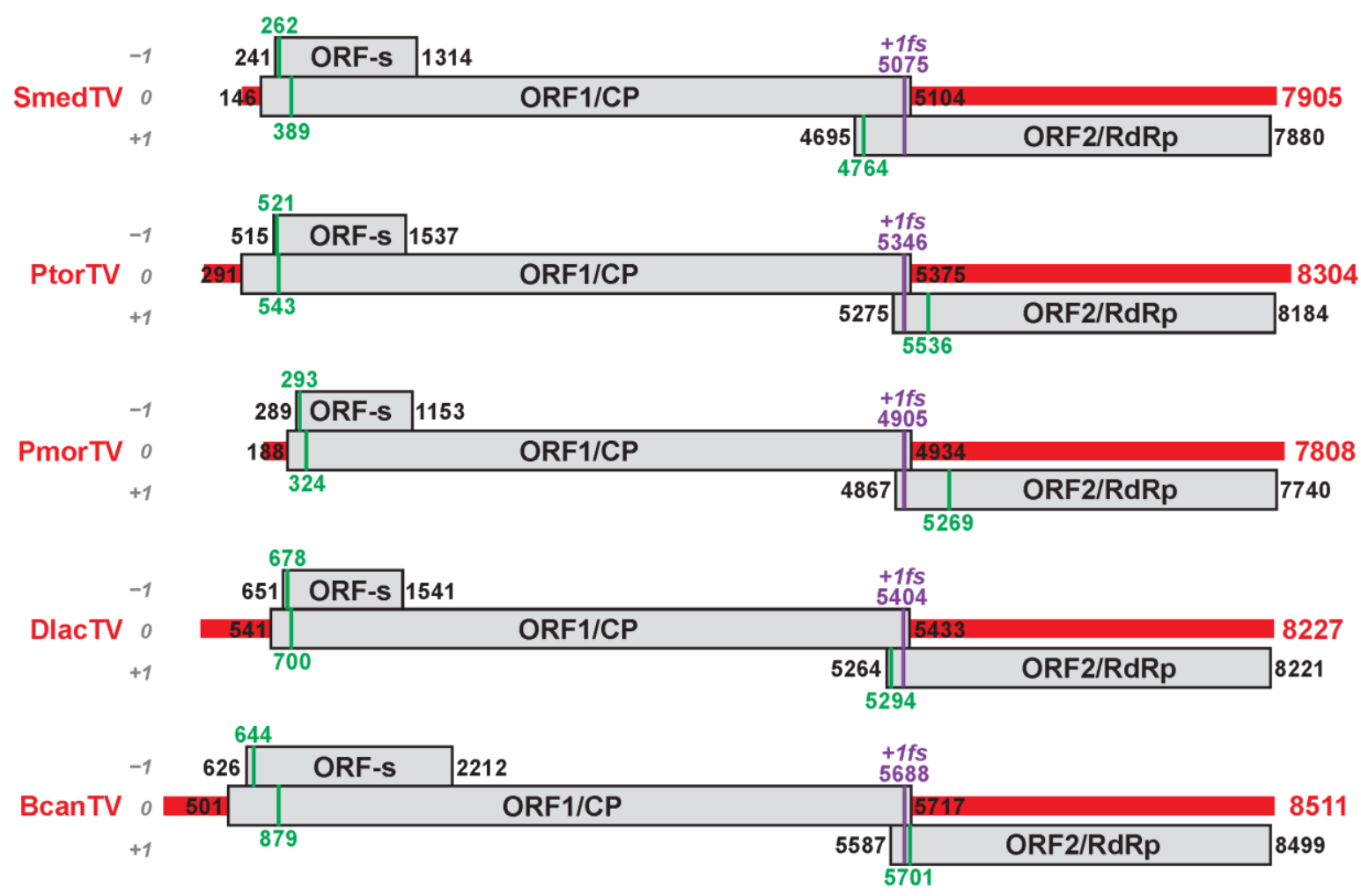

430 Figure 1: Scaled diagrams of apparent planarian virus genomes. Overall lengths of transcript

431 contigs are indicated at right. The genomic RNA plus strand of each virus is shown as a thick

432 horizontal red line. Long ORFs are shown as gray rectangles, labeled with the first and last nt

433 positions of each (including stop codons). The reading frame that includes ORF1 is defined as

434 frame 0, as labeled at left. The first in-frame AUG codon in each ORF is shown as a vertical green

435 line and labeled with the first nt position. The putative +1 ribosomal frameshifting site $(+1 f s)$ in

436 the region of ORF1-ORF2 overlap in each genome is also indicated. The diagrams for the 5 viruses

437 are aligned according to the position of the ORF1 stop codon. The diagram for SmedTV is that for

438 the consensus from the original TSA and PlanMine sequences (see text). 
bioRxiv preprint doi: https://doi.org/10.1101/551184; this version posted February 15,2019. The copyright holder for this preprint (which was not certified by peer review) is the author/funder, who has granted bioRxiv a license to display the preprint in perpetuity. It is made available under aCC-BY-NC-ND 4.0 International license.

Figure 2
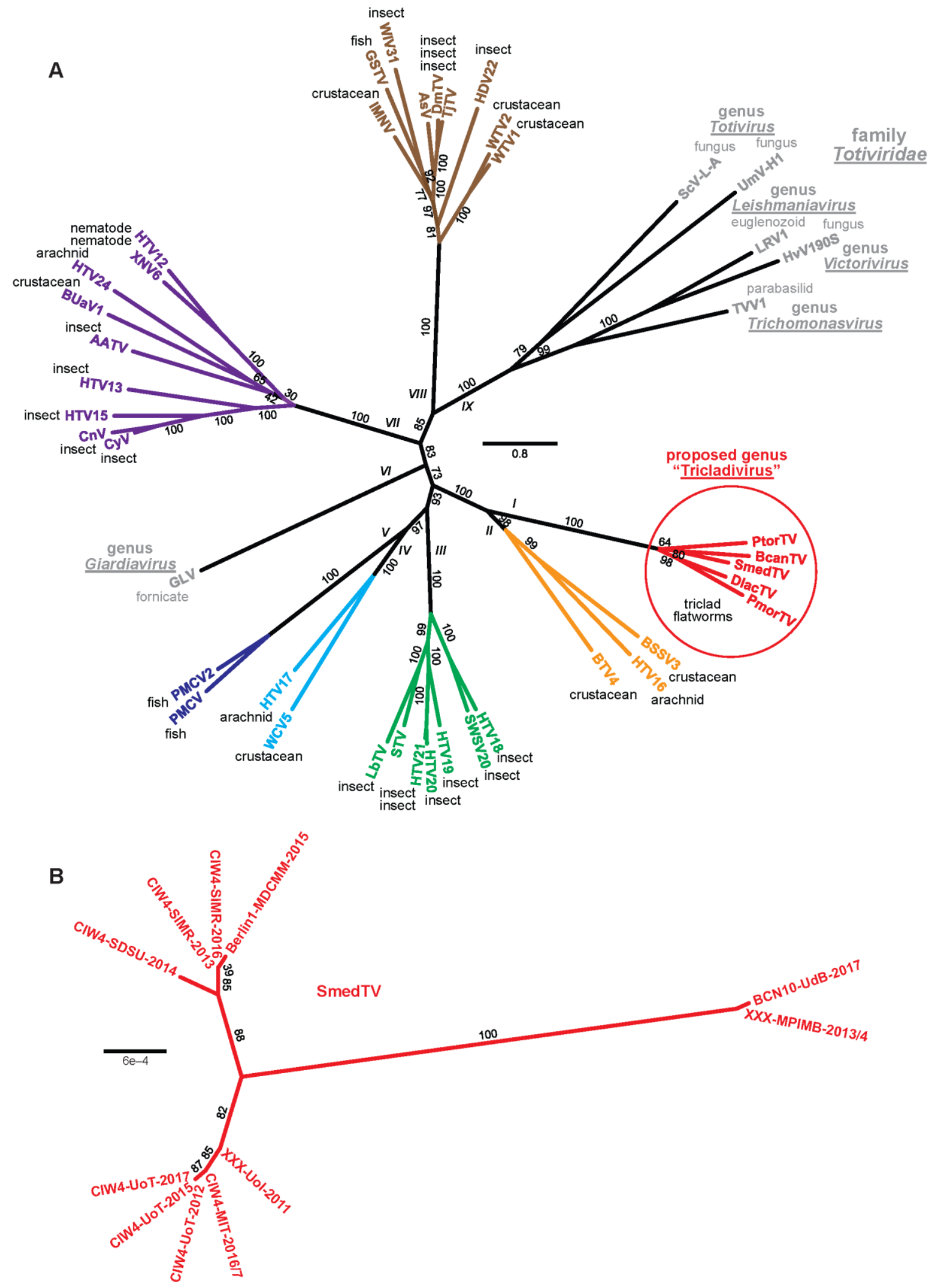
$442 \quad$ Figure 2: Unrooted radial phylograms. For each phylogram, aligned sequences were subjected to

443 maximum-likelihood phylogenetic analyses using ModelFinder, IQ-TREE, and UFBoot

444 (Trifinopoulos, Nguyen et al. 2016) as implemented with the "Find best and apply" option at

445 https://www.hiv.lanl.gov/content/ sequence/IQTREE/iqtree.html. Branch support values (from

4461000 bootstrap replicates) are shown in \% values. Scale bar indicates average number of

447 substitutions per alignment position. (A) Deduced amino acid sequences of the RdRp regions of

448 each virus were aligned using MAFFT 7.3 (E-INS-i). The following were found to apply by

449 ModelFinder: best-fit model according to BIC, LG+F+R6; model of rate heterogeneity, FreeRate

450 with 6 categories; site proportion and rates, $(0.0189,0.0067),(0.0433,0.1412),(0.1532,0.3344)$,

$451 \quad(0.2857,0.6616),(0.3798,1.2094)$, and $(0.1191,2.4694)$. See Table S2 for summary of virus names,

452 abbreviations, and RefSeq numbers. Different apparent monophyletic clades are labeled with

453 Roman numerals I-IX, and those constituted by viruses from apparent metazoan hosts are

454 differentially colored. Currently recognized members of family Totiviridae are labeled in gray. (B)

455 Nucleotide sequences of different SmedTV strains were aligned using MAFFT 7.3 (L-INS-i). The

456 following was found to apply by ModelFinder: best-fit model according to BIC, HKY+I. 
Figure 3

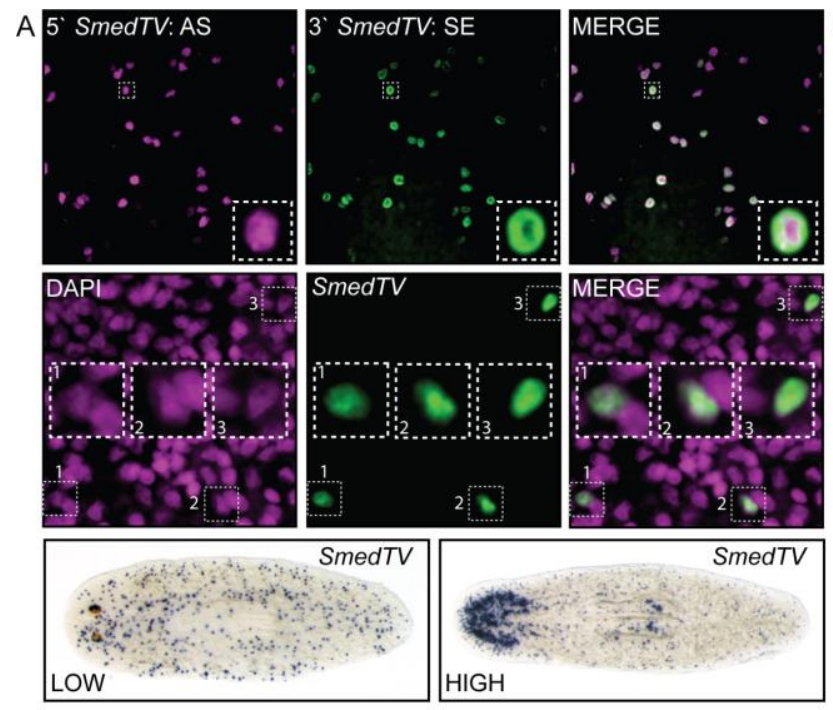

C
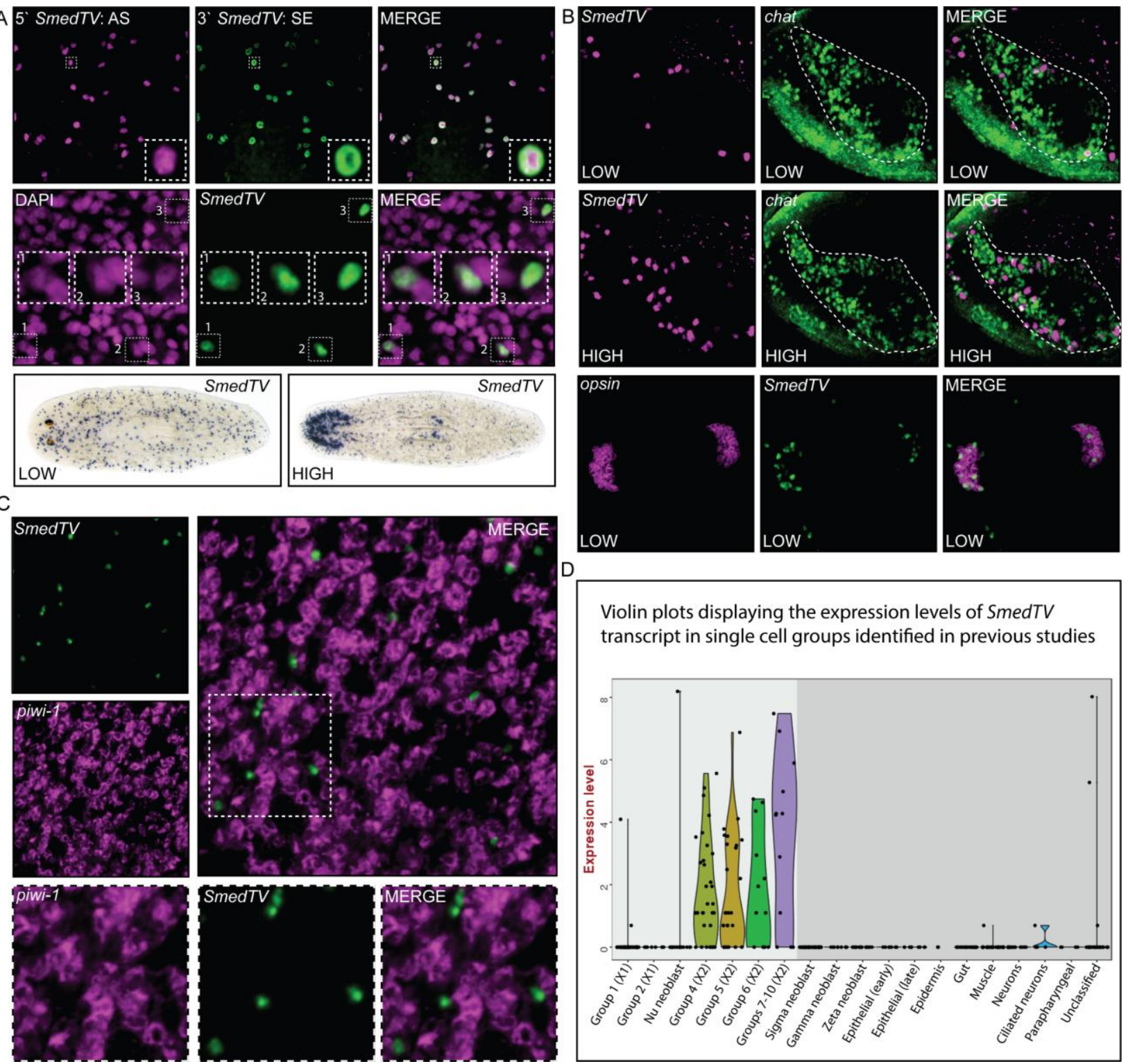

Figure 3: SmedTV expression is discrete and variable from worm to worm, cell type to cell type

A) Top: SmedTV antisense (AS) (magenta) and sense (SE) (green)

462 fluorescent RNA dFISH show perfect co-localization indicating the transcript is double stranded.

463 The boxed area is enlarged to show doubling. Middle: confocal image of FISH demonstrating the

464 nuclear localization of SmedTV (green) by doubling with nuclear stain dapi (magenta). The boxed

465 area is enlarged to show doubling. Bottom: WISH for SmedTV in wild-type intact animals showing 
466 discrete, but variable staining from worm to worm, cell type to cell type and within cell types.

467 Examples of "low" (left) and "high" (right) expression are shown. Note enrichment in neural

468 structures in "high" SmedTV expressing worms. B) dFISH confocal images illustrate SmedTV

469 expression in brain and eye spots. Top ("low" SmedTV expression) and middle ("high" SmedTV

470 expression): SmedTV expression (magenta) in 20 micron Z-stacks of cross-sectioned brain lobes

471 (taken just posterior to eye spots, enclosed within dotted line) defined by chat expression (green).

472 Note increase of SmedTV expressing cells within the brain lobes of worms from "high" SmedTV

473 cultures. Bottom: SmedTV (green) is also found at high levels with in eye-spots as defined by

474 photoreceptor marker opsin (magenta). C) SmedTV expression is absent from stem cell

475 populations as shown by lack of co-localization analysis through dFISH of SmedTV (green) and

476 piwi-1 (magenta). The boxed area is enlarged to show lack of doubling indicating that although in

477 close proximity the cells are distinct cells. D) Graphic illustration of previously published single

478 cell sequencing data represented in violin plots with SmedTV expression level on the Y-axis and

479 cell type on the x-axis. SmedTV expression is seen in multiple, but not all differentiated tissue

480 types. Data in lighter shaded area is from (Molinaro and Pearson 2016) (head enriched) and the

481 darker shaded area from (Wurtzel, Cote et al. 2015) (post pharyngeal). Note increased expression

482 seen in Pearson lab could be due to tissue specific or culture differences. All confocal image were

483 captured at $20 \times$ magnification. 
Figure 4
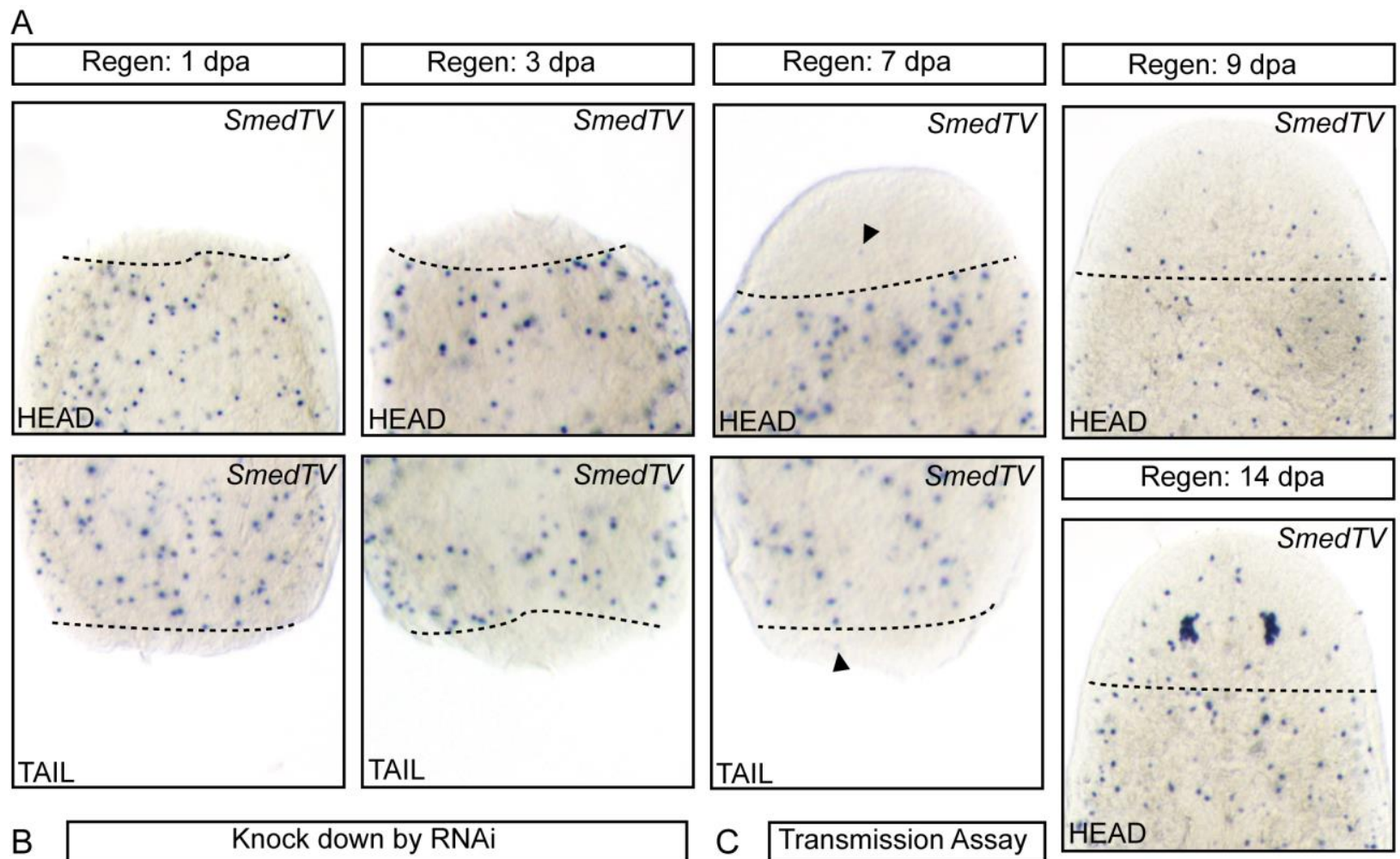

\section{B}
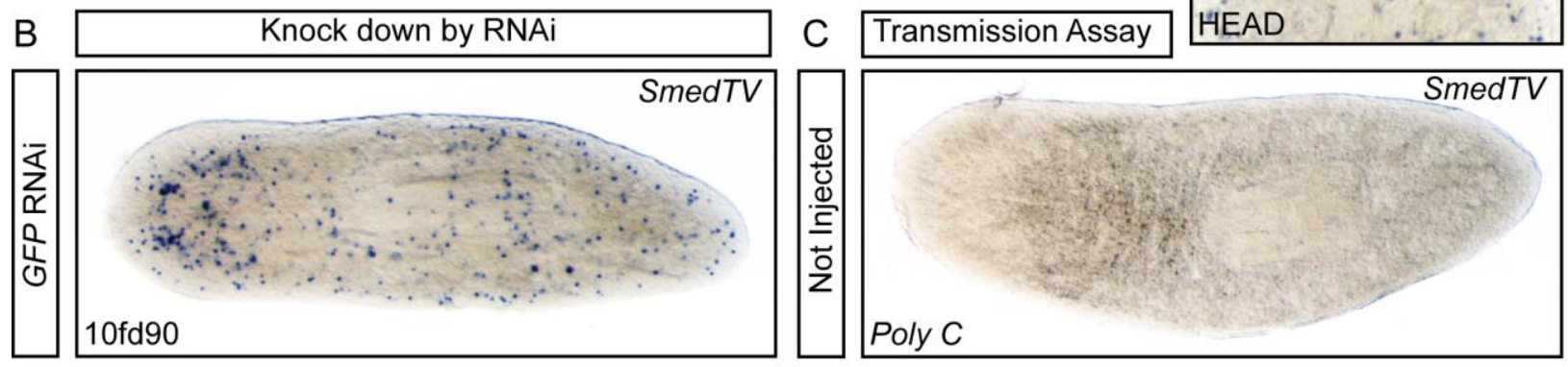

Transmission Assay

HEAD
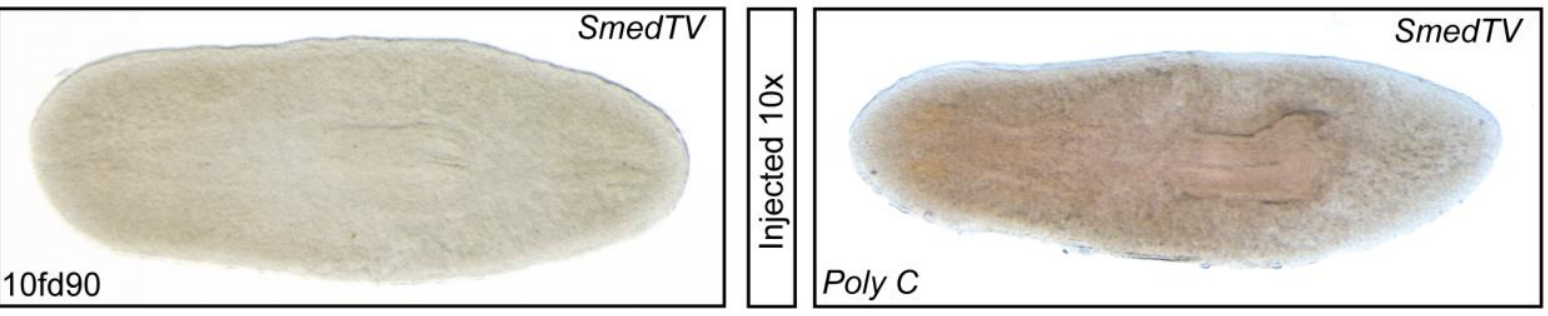

Figure 4: SmedTV expression dynamics. A) SmedTV expression, as seen by WISH in regenerating trunk fragments, is largely absent from the regenerating tissue until 9 days post amputation (top) with robust expression evident by $14 \mathrm{dpa}$ (bottom). Dotted line represents plane

B) WISH analysis of SmedTV 
bioRxiv preprint doi: https://doi.org/10.1101/551184; this version posted February 15,2019 . The copyright holder for this preprint (which was not certified by peer review) is the author/funder, who has granted bioRxiv a license to display the preprint in perpetuity. It is made available under aCC-BY-NC-ND 4.0 International license.

492 after 90 days. C) WISH analysis of SmedTV expression in Schmidtea polychroa following 10

493 injections of Smed CIW4 worm homogenate. Note expression is absent in both control and

494 injected worms.

495 


\section{$496 \quad$ Figure $\mathbf{S 1}$}

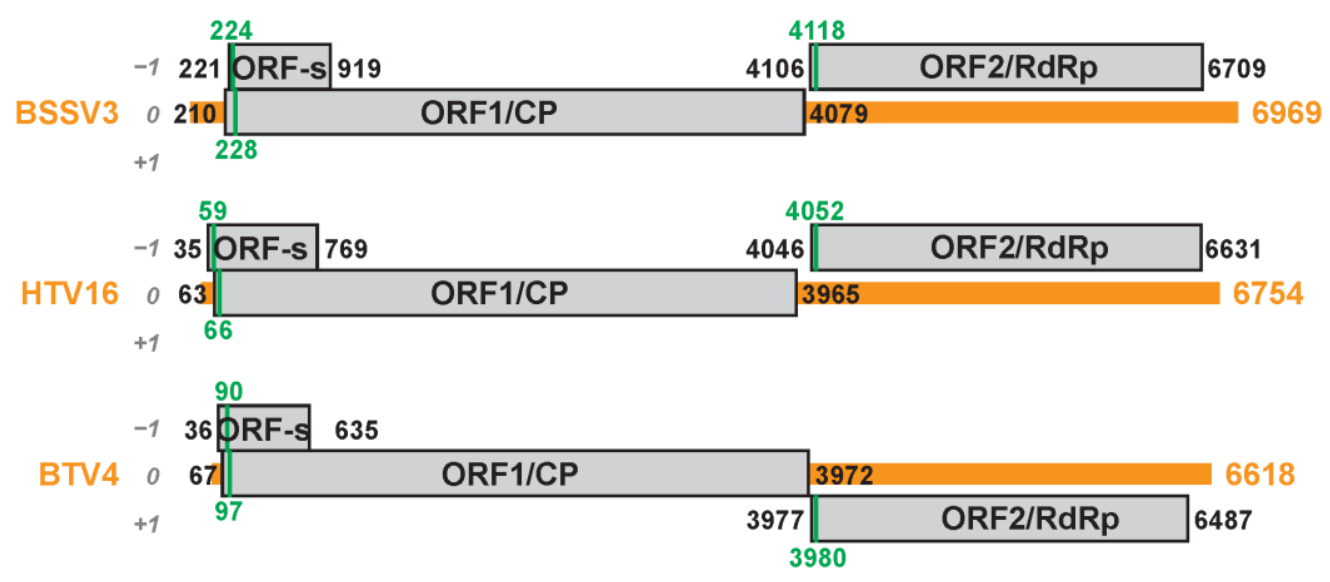

498 Figure S1: Scaled diagrams of putative arthropod virus genomes (clade most related to planarian

499 viruses in Fig. 2). Overall lengths of transcript contigs are indicated at right. The genomic RNA

500 plus strand of each virus is shown as a thick horizontal orange line. Long ORFs are shown as gray

501 rectangles, labeled with the first and last nt positions of each (including stop codons). The reading

502 frame that includes ORF1 is defined as frame 0, as labeled at left. The first in-frame AUG codon

503 in each ORF is shown as a vertical green line and labeled with the first nt position. The diagrams

504 for the 3 viruses are aligned according to the position of the ORF1 stop codon. 
Figure S2

\begin{tabular}{|c|c|c|c|c|c|c|c|c|c|}
\hline \multirow[t]{2}{*}{ P1IP2 } & & & \multicolumn{5}{|c|}{ Sequence identity between viruses (pairwise, \%): } & \multirow[b]{2}{*}{ BTV4 } & \multirow[b]{2}{*}{ HTV16 } \\
\hline & Viruses & BcanTV & DlacTV & PmorTV & PtorTV & SmedTV & BSSV3 & & \\
\hline & BcanTV & 100 & 37.7 & 35.7 & 38.0 & 42.6 & 21.3 & 21.5 & 21.5 \\
\hline & DlacTV & 22.1 & 100 & 41.7 & 36.0 & 41.6 & 19.3 & 17.9 & 21.2 \\
\hline & PmorTV & 22.4 & 36.1 & 100 & 35.5 & 37.4 & 20.6 & 21.2 & 22.2 \\
\hline & PtorTV & 23.3 & 26.0 & 25.1 & 100 & 38.3 & 20.4 & 19.1 & 19.6 \\
\hline & SmedTV & 23.3 & 28.9 & 26.7 & 28.9 & 100 & 20.2 & 19.4 & 20.7 \\
\hline & BSSV3 & 16.8 & 15.7 & 15.8 & 16.2 & 16.0 & 100 & 24.2 & 28.6 \\
\hline & BTV4 & 16.7 & 19.9 & 18.3 & 17.7 & 17.1 & 21.1 & 100 & 24.5 \\
\hline & HTV16 & 15.8 & 18.1 & 15.7 & 16.7 & 17.0 & 24.8 & 24.0 & 100 \\
\hline
\end{tabular}

\begin{tabular}{|c|c|c|c|c|c|c|c|c|}
\hline & & \multicolumn{9}{|c|}{ Sequence identity between viruses (pairwise, \%): } & & \\
\hline Viruses & BcanTV & DlacTV & PmorTV & PtorTV & SmedTV & BSSV3 & BTV4 & HTV16 \\
\hline BcanTV & 100 & $\mathbf{2 7 . 5}$ & $\mathbf{2 7 . 2}$ & $\mathbf{2 8 . 4}$ & $\mathbf{2 9 . 8}$ & $\mathbf{n r}$ & $\mathbf{n r}$ & $\mathbf{n r}$ \\
\hline DlacTV & $\mathbf{1 1 . 6}$ & 100 & $\mathbf{3 8 . 1}$ & $\mathbf{2 9 . 8}$ & $\mathbf{3 3 . 7}$ & $\mathbf{n r}$ & $\mathbf{n r}$ & $\mathbf{n r}$ \\
\hline PmorTV & $\mathbf{1 6 . 2}$ & $\mathbf{1 6 . 4}$ & 100 & $\mathbf{2 8 . 9}$ & $\mathbf{3 0 . 5}$ & $\mathbf{n r}$ & $\mathbf{n r}$ & $\mathbf{n r}$ \\
\hline PtorTV & $\mathbf{9 . 2}$ & $\mathbf{1 5 . 5}$ & $\mathbf{1 5 . 7}$ & 100 & $\mathbf{3 2 . 4}$ & $\mathbf{n r}$ & $\mathbf{n r}$ & $\mathbf{n r}$ \\
\hline SmedTV & $\mathbf{1 3 . 4}$ & $\mathbf{1 1 . 9}$ & $\mathbf{1 2 . 5}$ & $\mathbf{1 3 . 7}$ & 100 & $\mathbf{n r}$ & $\mathbf{n r}$ & $\mathbf{n r}$ \\
\hline BSSV3 & $\mathbf{9 . 5}$ & $\mathbf{1 8 . 1}$ & $\mathbf{1 4 . 8}$ & $\mathbf{4 . 4}$ & $\mathbf{1 0 . 6}$ & 100 & $\mathbf{n r}$ & $\mathbf{n r}$ \\
\hline BTV4 & $\mathbf{8 . 2}$ & $\mathbf{1 5 . 7}$ & $\mathbf{1 1 . 0}$ & $\mathbf{2 . 7}$ & $\mathbf{6 . 5}$ & $\mathbf{1 7 . 0}$ & 100 & $\mathbf{n r}$ \\
\hline HTV16 & $\mathbf{9 . 6}$ & $\mathbf{2 0 . 4}$ & $\mathbf{1 7 . 9}$ & $\mathbf{1 5 . 3}$ & $\mathbf{8 . 3}$ & $\mathbf{1 5 . 0}$ & $\mathbf{1 7 . 7}$ & 100 \\
\hline
\end{tabular}

Figure S2: Identity scores (\%) from global pairwise alignments of the deduced protein sequences.

509 Scores were determined using Needleall as implemented at http://www.bioinformatics.nl/ emboss-

511 panel, scores for P-s and P1+2 at lower left and upper right, respectively. Values are not shown

512 for P1+2 of BSSV3, BTV4, and HTV16 (nr, not relevant) because those viruses appear not to

513 express that fusion protein (P2 expressed as a separate protein). See text and Table S2 for virus

514 names, abbreviations, and accession numbers. Coloring is the same as in Fig. 2. 


\section{$516 \quad$ Figure S3}

\begin{tabular}{|c|c|c|c|c|c|c|c|c|c|c|c|}
\hline 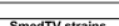 & $8+602011$ & & & SIWA & ucleotide mismatche & between SmedTV sti & ains (pairwise, number & & & & \\
\hline$\frac{\text { Smediv strains }}{X-U \text { ol-2011 }}$ & $x=01-2011$ & $\frac{C 1 W 4-001-2012}{3}$ & $\frac{C I W 4-001-2015}{3}$ & $\frac{\text { CIW4-UoT-2017 }}{3}$ & $\frac{\text { CIW4-MiT-2016/7 }}{2}$ & $\frac{\text { CIWA-SDSU-2014 }}{14}$ & \begin{tabular}{|l|} 
CIW4-SIMR-2013 \\
13
\end{tabular} & $\frac{\text { CIW4-SIMR-2016 }}{13}$ & Berlin1-MDCMM-2015 & BCN10-UdB-2017 & $\frac{\text { X-MPIMB-2013/4 }}{33}$ \\
\hline CIW4-UOT-2012 & & 0 & 0 & 0 & 1 & 17 & 14 & 14 & 15 & 33 & 34 \\
\hline CIW4-UOT-2015 & & & 0 & 0 & 1 & 17 & 14 & 14 & 15 & 33 & 34 \\
\hline CIW4-Uol-2017 & & & & & $\frac{1}{0}$ & $\frac{17}{16}$ & $\frac{14}{15}$ & 14 & $\frac{15}{16}$ & 33 & 34 \\
\hline CIW4-SDSU-2014 & & & & & & $\frac{0}{10}+5-50$ & $\frac{15}{5}$ & $\frac{10}{5}$ & $\frac{10}{6}$ & 38 & $\frac{33}{37}$ \\
\hline CIW4-SIMR-2013 & & & & & & & 0 & 0 & 1 & 35 & 36 \\
\hline CIW4-SIMR-2016 & & & & & & & & 0 & 1 & 35 & 36 \\
\hline Serlin1-MDCMM-201 & & & & & & & & & 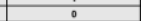 & 36 & 37 \\
\hline BCN10-UdB-2017 & & & & & & & & & & 0 & 1 \\
\hline X-MPIMB-2013/4 & & & & & & & & & & & 0 \\
\hline
\end{tabular}

518 Figure S3: Identity scores (\%) from global pairwise alignments of the nucleotide sequences for

519 different SmedTV strains. Scores were determined using Needleall as implemented at

520 http://www.bioinformatics.nl/ emboss-explorer/. SmedTV strain names include the specific $S$.

521 mediterranea strain name when annotated in the BioProject metadata (X if not clearly annotated),

522 followed by an abbreviation for the reporting institution, followed by the registration date for the

523 respective BioProject. 


\section{$525 \quad$ Figure S4}

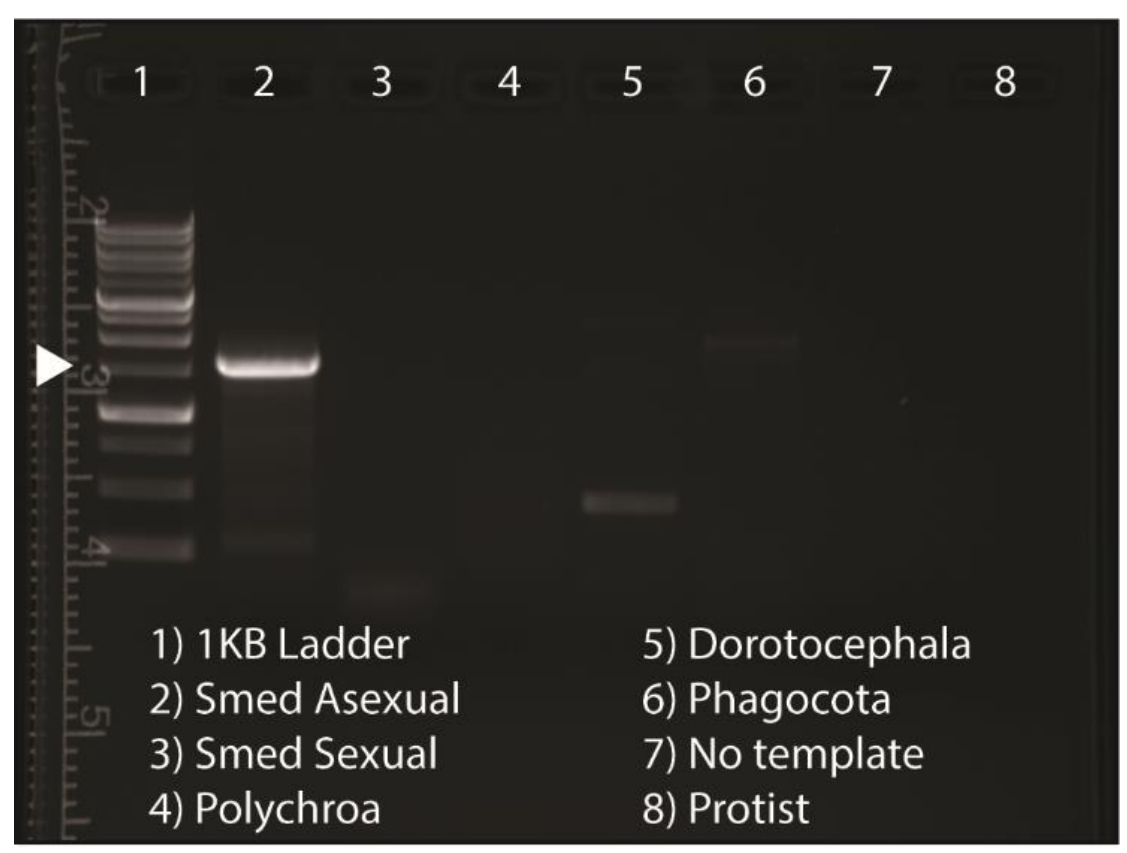

527 Figure S4: SmedTV transcript is unique to Smed Asexual planarians. Image of an EtBr agarose 528 gel under UV light following electrophoresis to visualize amplicons (or lack thereof) resulting 529 from 35 cycles of PCR using SmedTV specific primers (expected amplicon size of $\sim 1.5 \mathrm{~kb}$ ) on 530 cDNA generated from RNA isolated from multiple planarian species. Lane 1 is a DNA ladder. 531 The white arrowhead denotes the $1.5 \mathrm{~kb}$ band. Lanes 2-6 are for different planarian species as

532 labeled. A no template and protist (ubiquitous in planarian culture) cDNA controls are also 533 included. Note: a robust band is only observed in the Smed asexual sample. 
bioRxiv preprint doi: https://doi.org/10.1101/551184; this version posted February 15,2019 . The copyright holder for this preprint (which was not certified by peer review) is the author/funder, who has granted bioRxiv a license to display the preprint in perpetuity. It is made available under aCC-BY-NC-ND 4.0 International license.

Figure S5
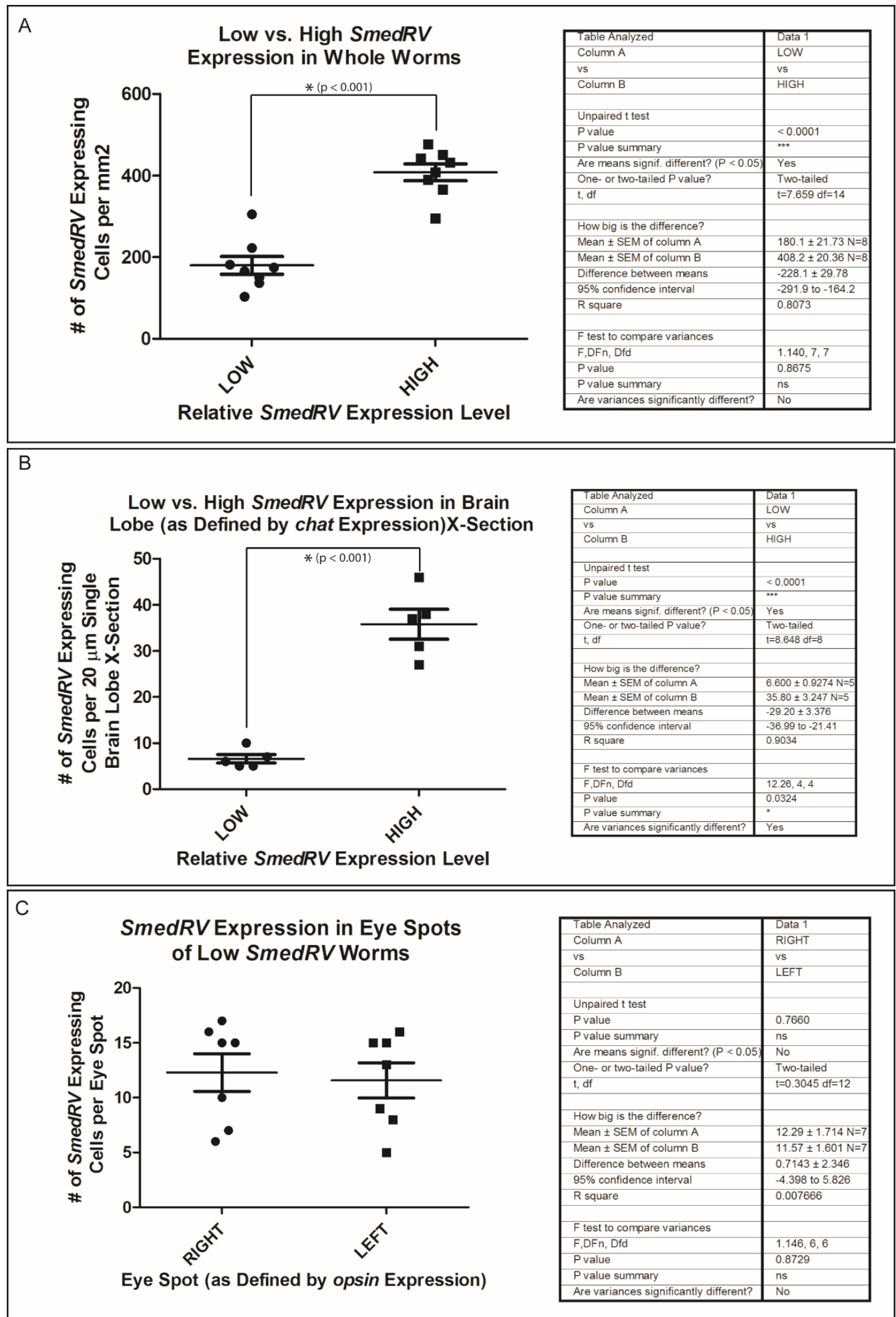
537 Figure S5: Graphical representation of quantification results of various SmedTV cell counts. A)

538 Number of SmedTV expressing cells per mm2 in worms from "low" and "high" SmedTV

539 expressing cultures. Note the significant increase in SmedTV expressing cell in "high" worms. A)

540 Number of SmedTV expressing cells per $20 \mu \mathrm{m}$ of cross-sectioned brain lobe (as defined by chat

541 expression) in worms from "low" and "high" SmedTV expressing cultures. Note the significant

542 increase in SmedTV expressing cells in "high" worms. C) Number of SmedTV expressing cells per

543 eye in wormsfrom "low" SmedTV expressing cultures. Error bars represent Standard Error of the

544 Mean (SEM). Note: statically analysis was done by Unpaired T-Test with data appearing in a table

545 to the right of each graph. 
bioRxiv preprint doi: https://doi.org/10.1101/551184; this version posted February 15,2019 . The copyright holder for this preprint (which was not certified by peer review) is the author/funder, who has granted bioRxiv a license to display the preprint in perpetuity. It is made available under aCC-BY-NC-ND 4.0 International license.

\section{$547 \quad$ Figure S6}
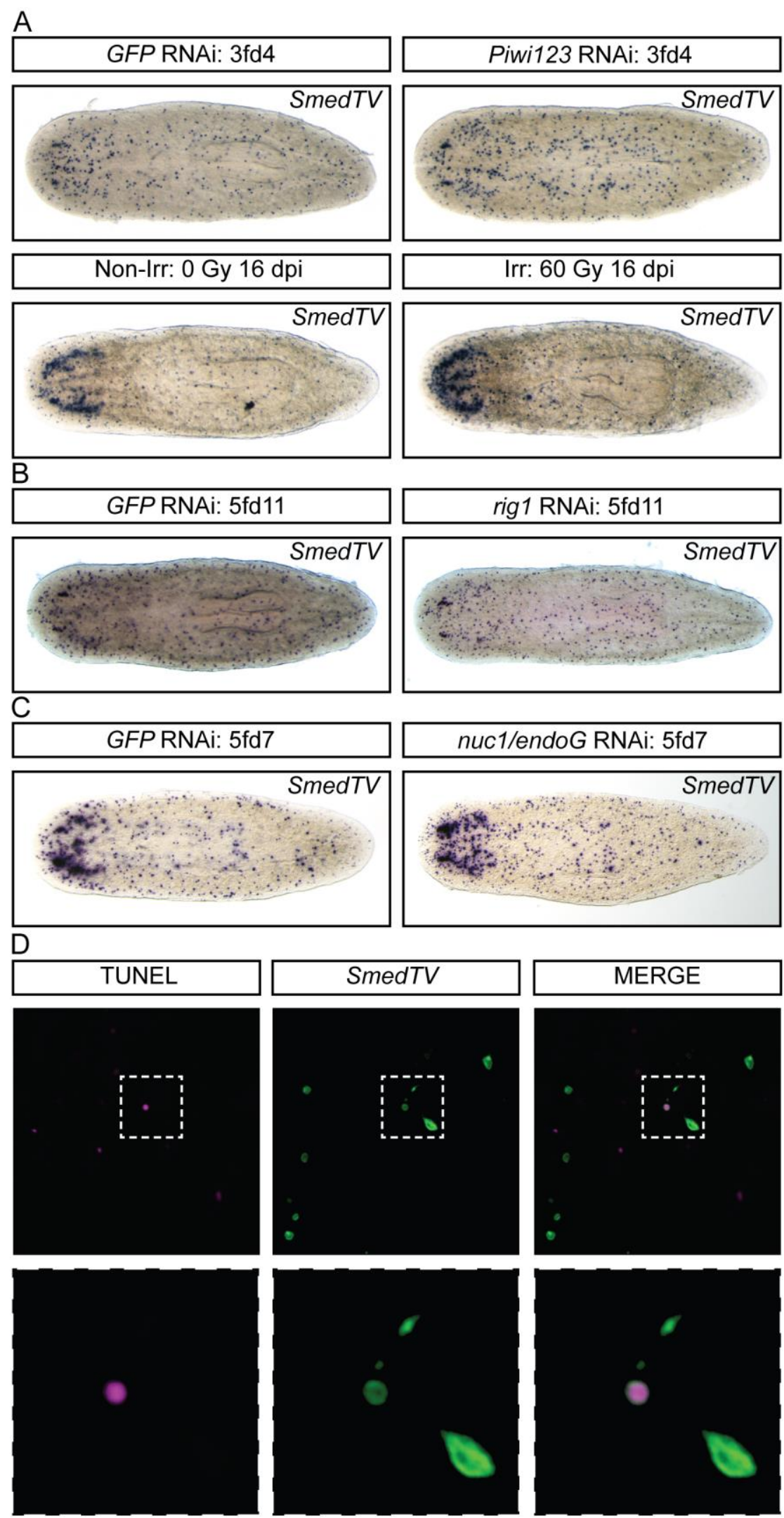
549 Figure S6: Non-regulators of SmedTV expression. WISH analysis of SmedTV expression

550 following different experimental conditions. A) Knockdown of stem cells by combining piwi1, 2

551 and 3 RNAi (top) or by lethal irradiation (bottom) has no effect on SmedTV expression. B) RNAi

552 knockdown of dsRNA helicase enzyme encoding gene, $R I G-1$, does not affect SmedTV expression.

553 C) RNAi knockdown of nuclease encoding gene nucl/endoG does not affect SmedTV expression. 


\section{Table 1. Flatworm toti-like viruses: coding features}

\begin{tabular}{|c|c|c|c|c|c|c|c|c|c|c|}
\hline Virus & $\begin{array}{l}\text { ORF-s } \\
\text { range } \\
\text { (nt) }\end{array}$ & $\begin{array}{l}\text { P-s: } \\
\text { length } \\
\text { (aa) }\end{array}$ & $\mathrm{pI}$ & $\begin{array}{l}\text { ORF1 } \\
\text { range } \\
\text { (nt) }\end{array}$ & $\begin{array}{l}\text { P1: } \\
\text { length } \\
\text { (aa) }\end{array}$ & $\mathrm{pI}$ & $\begin{array}{l}\text { ORF1+2 } \\
\text { range (nt) } \\
\text { (nt) }\end{array}$ & $\begin{array}{l}\mathrm{P} 1+2 \text { : } \\
\text { length } \\
\text { (aa) }\end{array}$ & $\mathrm{pI}$ & $\begin{array}{l}\text { Contig } \\
\text { length } \\
\text { (nt) }\end{array}$ \\
\hline SmedTV & $262-1317$ & 351 & 9.8 & $389-5107$ & 1572 & 6.1 & 389-5074:5076-7880 & 2496 & 8.0 & 7905 \\
\hline PtorTV & $521-1540$ & 339 & 7.1 & $543-5378$ & 1611 & 5.8 & $543-5345: 5347-8184$ & 2546 & 7.5 & 8304 \\
\hline PmorTV & $293-1156$ & 287 & 10.4 & $324-4937$ & 1537 & 5.9 & $324-4904: 4906-7740$ & 2471 & 6.8 & 7808 \\
\hline DlacTV & $678-1544$ & 288 & 10.3 & $700-5436$ & 1578 & 6.2 & $700-5403: 5405-8221$ & 2506 & 7.9 & 8227 \\
\hline BcanTV & $644-2218$ & 523 & 9.5 & $879-5723$ & 1613 & 6.5 & 879-5696:5698-8499 & 2539 & 8.1 & 8511 \\
\hline
\end{tabular}

Ranges include stop codon

SmedTV = consensus sequence from several transcriptomes (see text)

ORF $1+2=$ predicted product from +1 programmed ribosomal frameshifting in the ORF1-ORF2 overlap region

Table 2. Flatworm toti-like viruses: top hits to dsRNA viruses annotated as such in GenBank

\begin{tabular}{lllllll}
\hline \multicolumn{1}{c}{$\begin{array}{l}\text { P-s: } \\
\text { Virus }\end{array}$} & top hit & e-value & $\begin{array}{l}\text { P1: } \\
\text { top hit }\end{array}$ & E-value & $\begin{array}{l}\text { P2fs: } \\
\text { top hit }\end{array}$ & E-value \\
\hline SmedTV & nd & $>10$ & Piscine myocarditis-like virus & $1.5 \mathrm{e}-06$ & Leptopilina boulardi toti-like virus & $8.6 \mathrm{e}-09$ \\
PtorTV & nd & $>10$ & Piscine myocarditis virus & $1.5 \mathrm{e}-07$ & Leptopilina boulardi toti-like virus & $4.3 \mathrm{e}-12$ \\
PmorTV & nd & $>10$ & nd & $>10$ & Leptopilina boulardi toti-like virus & $1.0 \mathrm{e}-12$ \\
DlacTV & nd & $>10$ & Piscine myocarditis-like virus & $1.2 \mathrm{e}-04$ & Leptopilina boulardi toti-like virus & $1.9 \mathrm{e}-06$ \\
BcanTV & nd & $>10$ & Piscine myocarditis virus & 4 & Leptopilina boulardi toti-like virus & $5.4 \mathrm{e}-09$
\end{tabular}

Piscine myocarditis virus, AGA37470.1; Piscine myocarditis-like virus, YP_009229914.1; Leptopilina boulardi toti-like virus, YP_009072448.1 Ranges include stop codon

SmedTV $=$ consensus sequence from several transcriptomes (see text)

$\mathrm{ORF} 1+2=$ predicted product from +1 programmed ribosomal frameshifting in the ORF1-ORF2 overlap region 
Table S1. Flatworm toti-like viruses: top hits to all viruses annotated as such in GenBank

\begin{tabular}{lllllll}
\hline & P-s: & & P1: & & P2fs: \\
Virus & top hit & e-value & top hit & e-value & top hit & e-value \\
& & & & & & \\
\hline SmedTV & nd & - & Beihai toti-like virus 4 & $2.7 \mathrm{e}-15$ & Beihai toti-like virus 3 & $2.2 \mathrm{e}-52$ \\
PtorTV & nd & - & Piscine myocarditis virus & $4.8 \mathrm{e}-06$ & Beihai paphia shell virus 5 & $2.7 \mathrm{e}-43$ \\
PmorTV & nd & - & nd & - & Beihai toti-like virus 3 & $8.0 \mathrm{e}-52$ \\
DlacTV & nd & - & Beihai toti-like virus 4 & $2.7 \mathrm{e}-12$ & Wenzhou toti-like virus 2 & $4.2 \mathrm{e}-50$ \\
BcanTV & nd & - & nd & - & Beihai toti-like virus 3 & $1.4 \mathrm{e}-60$ \\
& & & & & & \\
\hline
\end{tabular}

Table S2. Other viruses used for phylogenetic analyses represented by Fig. 2

\begin{tabular}{llll} 
Clade label & Abbreviation & RefSeq number & Virus Name \\
\hline II & BSSV3 & NC_032536.1 & Behai sea slater virus 3 \\
& BTV4 & NC_032841.1 & Behai toti-like virus 4 \\
HTV16 & NC_032924.1 & Hubei toti-like virus 16 \\
III & LbTV & NC_025218.2 & Leptopolina boulardi toti-like virus \\
& HTV18 & NC_032948.1 & Hubei toti-like virus 18 \\
& HTV19 & NC_032424.1 & Hubei toti-like virus 19 \\
& HTV20 & NC_032806.1 & Hubei toti-like virus 20 \\
& HTV21 & NC_032819.1 & Hubei toti-like virus 21 \\
& SWSV20 & NC_032931.1 & Sanxia water strider virus 20 \\
& STV & NC_032851.1 & Shuangao toti-like virus \\
IV & HTV17 & NC_032942.1 & Hubei toti-like virus 17 \\
& WCV5 & NC_033022.1 & Wenzhou crab virus 5 \\
V & PMCV & NC_015639.1 & (Atlantic salmon) Piscine myocarditis virus \\
& PMCV2 & NC_029302.1 & (Golden shiner) Piscine myocarditis-like virus \\
GI & GLV & NC_003555.1 & Giardia lamblia virus \\
VII & CyV & NC_027212.1 & Camponotus yamaokai virus \\
& CnV & NC_029312.1 & Camponotus nipponicus virus \\
& AATV & NC_035674.1 & Australian Anopheles totivirus \\
& BUaV1 & NC_032746.1 & Beihai uca arcuata virus 1 \\
& & &
\end{tabular}


VIII

\begin{tabular}{|c|c|c|}
\hline HTV12 & NC_032831.1 & Hubei toti-like virus 12 \\
\hline HTV13 & NC_032878.1 & Hubei toti-like virus 13 \\
\hline HTV15 & NC_032919.1 & Hubei toti-like virus 15 \\
\hline HTV24 & NC_032938.1 & Hubei toti-like virus 24 \\
\hline XNV6 & NC_033719.1 & Xingshan nematode virus 6 \\
\hline IMNV & NC_007915.3 & (Penaeid shrimp) Infectious myonecrosis virus \\
\hline AsV & NC_014609.1 & Armigeres subalbatus virus SaX06-AK20 \\
\hline DmTV & NC_013499.1 & Drosophila melanogaster totivirus SW-2009a \\
\hline GSTV & NC_030295.1 & Golden shiner totivirus \\
\hline TjTV & NC_017084.1 & Tianjian totivirus \\
\hline HDV22 & NC_032902.1 & Hubei diptera virus 22 \\
\hline WIV31 & NC_033467.1 & Wuhan insect virus 31 \\
\hline WTV1 & NC_032462.1 & Wenzhou toti-like virus 1 \\
\hline WTV2 & NC_033012.1 & Wenling toti-like virus 2 \\
\hline ScV-L-A & NC_003745.1 & Saccharomyces cerevisiae virus L-A \\
\hline HvV190S & NC_003607.2 & Helminthosporium victoriae virus $190 \mathrm{~S}$ \\
\hline LRV1 & NC_002063.1 & Leishmania RNA virus $1-1$ \\
\hline TVV1 & NC_003824.1 & Trichomonas vaginalis virus 1 \\
\hline UmV-H1 & NC_003823.1 & Ustilago maydis virus $\mathrm{H} 1$ \\
\hline
\end{tabular}




\section{References}

556

557

558

559

560

561

562

563

564

565

566

567

568

569

570

571

572

573

574

575

576

577

578

579

580

Brandl, H., H. Moon, M. Vila-Farre, S. Y. Liu, I. Henry and J. C. Rink (2016). "PlanMine--a mineable resource of planarian biology and biodiversity." Nucleic Acids Res 44(D1): D764-773.

Cebria, F., T. Kudome, M. Nakazawa, K. Mineta, K. Ikeo, T. Gojobori and K. Agata (2002). "The expression of neural-specific genes reveals the structural and molecular complexity of the planarian central nervous system." Mech Dev 116(1-2): 199-204.

Currie, K. W., D. D. Brown, S. Zhu, C. Xu, V. Voisin, G. D. Bader and B. J. Pearson (2016). "HOX gene complement and expression in the planarian Schmidtea mediterranea." Evodevo 7: 7.

Gurley, K. A., J. C. Rink and A. Sánchez Alvarado (2008). "Beta-catenin defines head versus tail identity during planarian regeneration and homeostasis." Science 319(5861): 323-327.

Haugland, O., A. B. Mikalsen, P. Nilsen, K. Lindmo, B. J. Thu, T. M. Eliassen, N. Roos, M. Rode and O. Evensen (2011). "Cardiomyopathy syndrome of atlantic salmon (Salmo salar L.) is caused by a double-stranded RNA virus of the Totiviridae family." J Virol 85(11): 5275-5286.

Holmes, E. C. (2009). "RNA virus genomics: a world of possibilities." J Clin Invest 119(9): 24882495.

Kao, D., D. Felix and A. Aboobaker (2013). "The planarian regeneration transcriptome reveals a shared but temporally shifted regulatory program between opposing head and tail scenarios." BMC Genomics 14: 797.

Kell, A. M. and M. Gale, Jr. (2015). "RIG-I in RNA virus recognition." Virology 479-480: 110121.

Labbe, R. M., M. Irimia, K. W. Currie, A. Lin, S. J. Zhu, D. D. Brown, E. J. Ross, V. Voisin, G. D. Bader, B. J. Blencowe and B. J. Pearson (2012). "A comparative transcriptomic analysis reveals conserved features of stem cell pluripotency in planarians and mammals." Stem Cells 30(8): 17341745.

Lauter, G., I. Soll and G. Hauptmann (2011). "Two-color fluorescent in situ hybridization in the embryonic zebrafish brain using differential detection systems." BMC Dev Biol 11: 43. 
Liu, Y. X. and C. L. Dieckmann (1989). "Overproduction of yeast viruslike particles by strains deficient in a mitochondrial nuclease." Mol Cell Biol 9(8): 3323-3331.

Martinez, J., D. Lepetit, M. Ravallec, F. Fleury and J. Varaldi (2016). "Additional heritable virus

584 in the parasitic wasp Leptopilina boulardi: prevalence, transmission and phenotypic effects." J Gen

$585 \quad$ Virol 97(2): 523-535.

586 Merryman, M. S., A. S. Alvarado and J. C. Jenkin (2018). "Culturing Planarians in the 587 Laboratory." Methods Mol Biol 1774: 241-258.

588 Molinaro, A. M. and B. J. Pearson (2016). "In silico lineage tracing through single cell 589 transcriptomics identifies a neural stem cell population in planarians." Genome Biol 17: 87.

590 Newmark, P. A., P. W. Reddien, F. Cebria and A. Sanchez Alvarado (2003). "Ingestion of 591 bacterially expressed double-stranded RNA inhibits gene expression in planarians." Proc Natl 592 Acad Sci U S A 100 Suppl 1: 11861-11865.

593 Newmark, P. A. and A. Sanchez Alvarado (2002). "Not your father's planarian: a classic model 594 enters the era of functional genomics." Nat Rev Genet 3(3): 210-219.

595 Ozata, D. M., I. Gainetdinov, A. Zoch, D. O'Carroll and P. D. Zamore (2019). "PIWI-interacting 596 RNAs: small RNAs with big functions." Nat Rev Genet 20(2): 89-108.

597 Palakodeti, D., M. Smielewska, Y. C. Lu, G. W. Yeo and B. R. Graveley (2008). "The PIWI 598 proteins SMEDWI-2 and SMEDWI-3 are required for stem cell function and piRNA expression 599 in planarians." RNA 14(6): 1174-1186.

600 Pearson, B. J., G. T. Eisenhoffer, K. A. Gurley, J. C. Rink, D. E. Miller and A. Sanchez Alvarado 601 (2009). "Formaldehyde-based whole-mount in situ hybridization method for planarians." Dev Dyn $602 \quad 238(2): 443-450$.

603 Pellettieri, J. and A. S. Alvarado (2007). "Cell turnover and adult tissue homeostasis: from humans 604 to planarians." Annu Rev Genet 41: 83-105. 
605

606

607

608

609

610

611

612

613

614

615

616

617

618

619

620

621

622

623

624

625

626

627

628

629

630
Ramm, S. A. (2017). "Exploring the sexual diversity of flatworms: Ecology, evolution, and the molecular biology of reproduction." Mol Reprod Dev 84(2): 120-131.

Rebrikov, D. V., E. A. Bogdanova, M. E. Bulina and S. A. Luk'ianov (2002). "[A new planarian extrachromosomal virus-like element revealed by subtraction hybridization]." Mol Biol (Mosk) 36(6): 1002-1011.

Rebrikov, D. V., M. E. Bulina, E. A. Bogdanova, L. L. Vagner and S. A. Lukyanov (2002). "Complete genome sequence of a novel extrachromosomal virus-like element identified in planarian Girardia tigrina." BMC Genomics 3: 15.

Reddien, P. W., N. J. Oviedo, J. R. Jennings, J. C. Jenkin and A. Sanchez Alvarado (2005). "SMEDWI-2 is a PIWI-like protein that regulates planarian stem cells." Science 310(5752): 13271330.

Reddien, P. W. and A. Sanchez Alvarado (2004). "Fundamentals of planarian regeneration." Annu

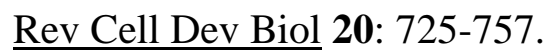

Roossinck, M. J. (2017). "Deep sequencing for discovery and evolutionary analysis of plant viruses." Virus Res 239: 82-86.

Rozanski, A., H. Moon, H. Brandl, J. M. Martin-Duran, M. A. Grohme, K. Huttner, K. Bartscherer, I. Henry and J. C. Rink (2019). "PlanMine 3.0-improvements to a mineable resource of flatworm biology and biodiversity." Nucleic Acids Res 47(D1): D812-D820.

Saberi, A., A. A. Gulyaeva, J. L. Brubacher, P. A. Newmark and A. E. Gorbalenya (2018). "A planarian nidovirus expands the limits of RNA genome size." PLoS Pathog 14(11): e1007314.

Shi, M., X. D. Lin, J. H. Tian, L. J. Chen, X. Chen, C. X. Li, X. C. Qin, J. Li, J. P. Cao, J. S. Eden, J. Buchmann, W. Wang, J. Xu, E. C. Holmes and Y. Z. Zhang (2016). "Redefining the invertebrate RNA virosphere." $\underline{\text { Nature. }}$

Solana, J., D. Kao, Y. Mihaylova, F. Jaber-Hijazi, S. Malla, R. Wilson and A. Aboobaker (2012). "Defining the molecular profile of planarian pluripotent stem cells using a combinatorial RNAseq, RNAi and irradiation approach." Genome Biol 13(3): R19. 
631 Trifinopoulos, J., L. T. Nguyen, A. von Haeseler and B. Q. Minh (2016). "W-IQ-TREE: a fast

632 online phylogenetic tool for maximum likelihood analysis." Nucleic Acids Res 44(W1): W232-

633235.

634 Verneau, O., L. Du Preez and M. Badets (2009). "Lessons from parasitic flatworms about 635 evolution and historical biogeography of their vertebrate hosts." C R Biol 332(2-3): 149-158.

636 Wagner, D. E., I. E. Wang and P. W. Reddien (2011). "Clonogenic neoblasts are pluripotent adult 637 stem cells that underlie planarian regeneration." Science 332(6031): 811-816.

638 Wurtzel, O., L. E. Cote, A. Poirier, R. Satija, A. Regev and P. W. Reddien (2015). "A Generic and 639 Cell-Type-Specific Wound Response Precedes Regeneration in Planarians." Dev Cell 35(5): 632640645.

641 Zhu, S. J., S. E. Hallows, K. W. Currie, C. Xu and B. J. Pearson (2015). "A mex3 homolog is 642 required for differentiation during planarian stem cell lineage development." Elife 4.

643 Zhu, S. J. and B. J. Pearson (2016). "(Neo)blast from the past: new insights into planarian stem 644 cell lineages." Curr Opin Genet Dev 40: 74-80. 